\title{
Time Reversal in Subwavelength-Scaled Resonant Media: Beating the Diffraction Limit
}

\author{
Fabrice Lemoult, Abdelwaheb Ourir, Julien de Rosny, Arnaud Tourin, Mathias Fink, \\ and Geoffroy Lerosey
} Institut Langevin, Ecole Supérieure de Physique et de Chimie Industrielle de la Ville de Paris, UMR CNRS 7587, 10 Rue Vauquelin,
75005 Paris, France

Correspondence should be addressed to Geoffroy Lerosey, geoffroy.lerosey@espci.fr

Received 14 December 2010; Revised 5 April 2011; Accepted 15 June 2011

Academic Editor: M. C. E. Yagoub

Copyright ( $\odot 2011$ Fabrice Lemoult et al. This is an open access article distributed under the Creative Commons Attribution License, which permits unrestricted use, distribution, and reproduction in any medium, provided the original work is properly cited.

Time reversal is a physical concept that can focus waves both spatially and temporally regardless of the complexity of the propagation medium. Time reversal mirrors have been demonstrated first in acoustics, then with electromagnetic waves, and are being intensively studied in many fields ranging from underwater communications to sensing. In this paper, we will review the principles of time reversal and in particular its ability to focus waves in complex media. We will show that this focusing effect depends on the complexity of the propagation medium rather than on the time reversal mirror itself. A modal approach will be utilized to explain the physical mechanism underlying the concept. A particular focus will be given on the possibility to break the diffraction barrier from the far field using time reversal. We will show that finite size media made out of coupled subwavelength resonators support modes which can radiate efficiently in the far field spatial information of the near field of a source. We will show through various examples that such a process, due to reversibility, permits to beat the diffraction limit using far field time reversal, and especially that this result occurs owing to the broadband inherent nature of time reversal.

\section{Introduction}

The reversibility of the equations governing the propagation of waves, whether acoustic or electromagnetic, is of major interest in many fields of wave physics. This property is quite intriguing on a fundamental point of view but has also led to many fascinating discoveries within the last years. Among them, time reversal (TR) has been a major subject of studies in various fields such as ultrasound acoustics, seismology, microwave, or more recently in the optical domain.

In a typical TR experiment, a source emits a short pulse in a medium, which generates a wavefield that propagates away from it. Then this wavefield is measured onto a set of location on an array of sensors, the so-called time reversal mirror (TRM). The measured signals are digitized, memorized, and flipped in time. This first step, known as the "learning step", results in the knowledge of a set of impulse responses between the source and the TRM, which are the broadband equivalents of the Green's functions. In the second step, the time reversal one, these time reversed signals are sent back in the medium by the sensors, which results in a spatiotemporal focusing of the generated wavefield onto the initial source position, and at a specific time named the "collapse time" [1-3].

It has been shown that the temporal focusing of the wave depends on the medium as well as on the bandwidth used and the number of sensors constituting the TRM [24]. On a spatial point of view, in a homogeneous medium, time-reversed waves converge toward the focal point on a spot whose width is limited by the numerical aperture of the TRM, similar to the way conventional lenses focus light $[1,5]$. In heterogeneous media, however, TRM behaves very differently than regular lenses, and it has been proved that the numerical aperture of the TRM does not play any role when the complexity of the medium is increased [2]. In the extreme case of TR in a chaotic closed cavity, it has even been shown that a single channel TRM can focus waves down to the size 
of half a wavelength, that is, the diffraction limit, provided that broadband signals be used [6].

TR, which was first demonstrated with ultrasounds, has been studied quite intensively in acoustic, and has led to interesting concepts such as TR underwater communications [7], interactive objects [8] or acoustic bazookas [9]. Then, the concept was transposed to the electromagnetic part of the spectrum, and it was shown that TR experiments can be done at very high frequency using modulated signals, since in this case only the low frequency complex envelope of the signals has to be time reversed $[10,11]$. Since then, TR of electromagnetic waves has been studied in various areas such as sensing, detection and imaging [12-15], wireless communications [16-19], or medical imaging and therapy [20, 21].

One of the most astonishing results obtained using TR of electromagnetic waves is the fact that one can break the diffraction barrier using a TRM and a microstructured material, or in other words, that one can focus electromagnetic waves on deep subwavelength spots and from the far-field using TR [22-24]. This opens up very promising avenues in various fields such as telecommunications [18], imaging [20], or therapy [21] in microwaves, but also lithography and sensing at higher frequencies $[25,26]$.

The goal of this paper is to review the most recent works dealing with subwavelength focusing using TR, to explain this concept, generalize this approach, and propose new media for this purpose at the light of the proposed theory. To that aim, we will explain in a first part the basic principles of TR of waves, and in particular we will show that the broadband nature of TR experiments is of fundamental interest, hence distinguishing it from phase conjugation methods. We will also in this part transpose the concept to the specific case of electromagnetic waves, demonstrate analytically the result of a TR focusing experiment, and review some experimental results obtained in homogeneous media. Then, we will mention the original experiments that proved the possibility to focus electromagnetic waves on a deep subwavelength scale inside a random collection of metallic scatterers using far-field TR. A link will be made with the theory of TR focusing, and a theoretical study will be proposed that explains those results in terms of subwavelength Bloch modes in periodic arrays of subwavelength resonators. Finally in the last part we will present new results of TR subwavelength focusing from the far-field in arrays of split ring resonators, link those results with the developed approach, and generalize the concept to other possible structures.

\section{Basic Principles of Time Reversal Focusing}

The principle of TR is based on the fact that if within a given medium there exists a solution of the wave equation denoted $S(t)$, then another dual solution, namely, the time reversed one $S(-t)$, necessarily exists as well. In other words, if one is able to record the entire field created by a diverging source placed inside a reversible medium, time reversing and reemitting the latter generates a wavefield that converges toward the initial source. Such an ideal TR experiment, however, requires the knowledge of the field throughout the medium, which is impossible in most practical environments. In practice, TR is made possible thanks to the Helmholtz-Kirchoff integral theorem. The latter states that the knowledge of a wavefield on the boundaries of a closed surface holds that of the field inside the whole volume contained by the surface. Hence, a more realistic TR experiment can be imagined as follows (Figure 1): a source generates a brief pulse inside a heterogeneous medium that is surrounded by sensors which record the corresponding fields until all the energy has exited the closed surface. In a second step, the sensors play the recorded fields in a reversed chronology which generates the time-reversed wavefield on the boundary of the closed surface. This conceptual TR experiment, named the "Time Reversal Cavity", has been theoretically studied in acoustics $[27,28]$. It has been shown that it produces at a point $r$ and a given frequency a field that is proportional to the imaginary part of the Green's function between the initial source point $r_{0}$ and $r$.

$$
\phi_{\mathrm{TR}}(r) \propto \operatorname{Im}\left(G\left(\omega, r_{0}, r\right)\right) .
$$

Covering a whole surface with a collections of sensors respecting the Nyquist criterion, though, requires an enormous amount of electronic and is evidently not suitable in a regular experiment. In practice, closed TRMs are difficult to realize and the TR operation is usually performed on a limited angular area, thus apparently limiting focusing quality. A TRM consists typically of a small number of elements or time reversal channels. The major interest of TRM, compared to classical focusing devices (lenses and beam forming), is certainly the relation between the medium complexity and the size of the focal spot. A TRM acts as an antenna that uses complex environments to appear wider than it is, resulting in a refocusing quality that does not depend on the TRM aperture.

The reference experiment using small aperture TRM is the following [2-4]: a multiple scattering medium is placed between a point-like sensor and a TRM which are separated by a distance much larger than a wavelength (Figure 2(a)). A short pulse is emitted by the point source, and the set of impulse response from this point to the array of sensors constituting the TRM is acquired. Those responses are then flipped in time and sent back by the TRM acting now as a source thanks to reciprocity. It is then possible to scan the field produced by this TR focusing by translating the point source sensor parallel to the exit of the multiple scattering media. In doing so, one obtains a focal spot whose width is not limited by the numerical aperture of the TRM, but rather by that of the multiple scattering which can be much larger. The temporal and spatial signal to noise ratios of such focusing experiments have been shown to depend linearly on the total number of degrees of freedom of the medium, namely, the number of uncorrelated sensors in the array times the number of uncorrelated frequency in the bandwidth [2,3]. Very interestingly, the optics community has realized only recently that such experiments can be per- 


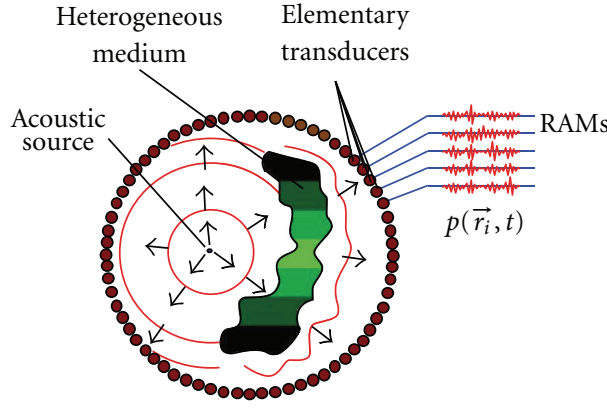

(a)

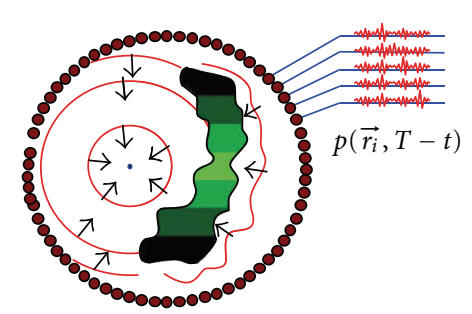

(b)

FIGURE 1: (a) Recording step: a closed surface is filled with transducer elements. A point-like source generates a wave front which is distorted by heterogeneities. The distorted pressure field is recorded on the cavity elements. (b) Time-reversed or reconstruction step: the recorded signals are time-reversed and reemitted by the cavity elements. The time-reversed pressure field backpropagates and refocuses exactly on the initial source.

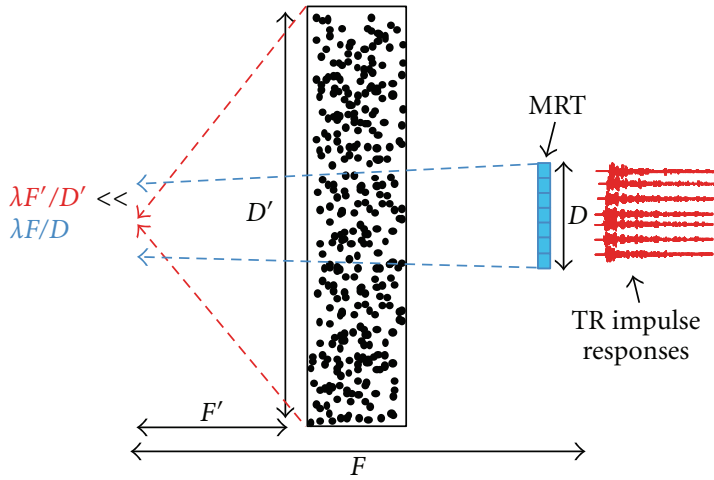

(a)

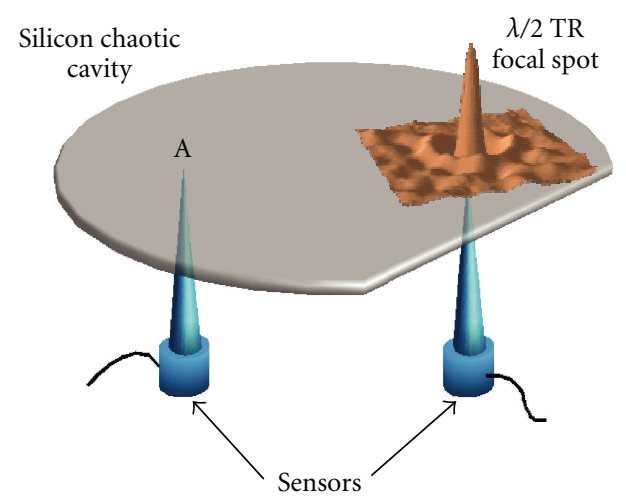

(b)

FIgURE 2: (a) Typical focusing experiment using TR. A multiple scattering medium is placed between the TRM and a point-like sensor. The size of the focal spot obtained with TR is no longer limited by the aperture of the TRM but rather by that of the multiple scattering medium. (b) Simplified view of a single channel TR focusing experiment realized in a silicon cavity. The field produced by TR at the collapse time is scanned using a heterodyne interferometer, and its map is superimposed on the cavity: a diffraction limited spot is obtained using only one emitter.

formed in the visible range, by means of spatial light modulators and in a monochromatic regime. Focusing well below the Rayleigh limit of conventional lenses has been demonstrated by inserting a multiple scattering media (a layer of paint) between an SLM and a CCD camera using this concept [29-31].

What happens when the number of sensors of the TRM is reduced down to a single one? Regarding the mentioned optical experiments, the answer is quite obvious: a single laser source that is shined through a multiple scattering medium gives rise to a so-called speckle pattern, that is, a random interference pattern. It is clear that no focusing can occur whatsoever in this case since changing the phase of this single source only changes the phase of the output speckle, hence precluding any focusing effect. The solution to this problem lies in the use of interference between statistically independent speckles or equivalently between speckle patterns at frequencies which are uncorrelated. This is where TR strongly differs from its monochromatic ancestor, namely, phase conjugation. In fact, our students in acoustic waves realize this everyday experiment during their practical classes: a single sensor is used as the TRM and a multiple scattering medium is placed between the latter and a movable wavelength large sensor. After TR, they can observe that the waves are tightly focused on the original source position with a spot size limited by the diffraction when the numerical aperture of the multiple scattering medium is larger than unity.

This very simple yet fascinating experiment is easier to understand considering the setup of Figure 2(b) taken from [6]. An aluminium cone coupled to a transducer generated waves at one point (A) of a nonsymmetrical and ergodic silicon cavity. A second transducer is used as a receiver. The central frequency of the transducers is set to $1 \mathrm{MHz}$ and their relative bandwidth to $100 \%(\omega=1 \mathrm{MHz})$. The source is considered point-like and isotropic because the cone tip is much smaller than the central wavelength. A heterodyne laser interferometer measures the displacement field as a function of time at different points on the cavity, resulting in a spatiotemporal mapping of the field propagating in the 
cavity. In the learning step, the impulse response is acquired between points $A$ and $B$, digitized and time reversed. Then the time reversed signal is sent from point $\mathrm{A}$ and the field generated by TR is scanned using the interferometer around point $B$. At the collapse time, the field, represented in the figure, is focused onto a spot whose width is about half a wavelength, limited by the diffraction limit. In other words, in this cavity, it is possible to reach the diffraction limit using a single sensor, thanks to TR.

This can actually be explained through a simple physical picture. Such a cavity can indeed be described in terms of modes, which are eigenmodes of the propagation and whose eigenvalues are their resonant frequencies. When a point source emits a short pulse in this medium (at point A), it excites all the modes that are nonzero at this location, each one at its resonant frequency, just as a guitar cord being slapped. Measuring the field at point B then simply consists in acquiring the phase and amplitude of the relative eigenmodes of the cavity at this specific position, at every resonance frequency, and with the condition that those modes are all in phase in A. The TR focusing therefore naturally comes out considering that time reversing an impulse response is equivalent to phase conjugating the signal at every frequency of the bandwidth: $S(t)$ to $S(-t) \Leftrightarrow$ $S(\omega)$ to $S^{*}(\omega)$. Indeed, sending the time-reversed impulse response from point $A$ annuls the phase of each eigenmode at point $\mathrm{B}$ and insures that all eigenmodes within the bandwidth interfere constructively at this point and at a specific time, the collapse time. From this very simple approach, one can also infer the signal to noise ratio of this focusing operation, namely, the ratio of the TR peak energy to the standard deviation of the field measure away from $\mathrm{B}$ and at a time different than the collapse one; at this specific time and at point $\mathrm{B}$, all the modes interfere coherently, everywhere else and at any other time they add up incoherently: the SNR roughly equals the number of eigenmodes of the cavity within the bandwidth. Of course focal spot therefore has a width, which is given by the average correlation length of the modes, around half of the central wavelength, since within a disk of diameter $\lambda / 2$ around $\mathrm{B}$, all points are in phase with $B$ at every time.

At this point, it is worth making the link between this modal approach and the results of the time reversal cavity [27] linking the TR produced field to the imaginary part of the Green's functions of the medium. This result, independent of the complexity of the medium, was a monochromatic one which can easily be integrated over the bandwidth in order to obtain the spatially varying field after TR and at the collapse time:

$$
\phi_{\mathrm{TR}}(r, t=0) \propto \int_{\text {Bandwidth }} \operatorname{Im}\left(G\left(r_{0}, r, \omega\right)\right) d \omega .
$$

Within a cavity or a complex medium where a modal analysis can be used, and if there is no degenerated modes which is the case for a high enough quality factor chaotic cavity, Green's functions are proportional to the eigenmodes. Hence integrating (1) is formally equivalent to adding the eigenmodes of the cavity that resonate in the bandwidth.
From now on, we will concentrate on the case of time reversal of electromagnetic waves.

Electromagnetic waves differ from acoustic ones since they are polarized, like elastic waves. Therefore, one has to take into account the orientation of the sources. In a typical time reversal scheme, a dipole source oriented along a given axis emits a short pulse. The wavefield propagates and is recorded with one antenna or a set of antennas, namely, the time reversal mirror. Second, the recorded signals are digitized, flipped in time, and transmitted back by the same set of antennas. An ideal TRM, "an electromagnetic time reversal cavity", should cover a closed surface that totally surrounds the source, with three antennas every $(\lambda / 2)^{2}$, each one being sensible to one polarization of the electromagnetic field. Such a cavity would then grant the knowledge of the electromagnetic field inside the whole volume contained in the closed surface, since HelmholtzKirchoff still holds. Due to the fact that the propagation equations for electromagnetic waves are also reversible, if one could realize such a time reversal experiment, the generated wavefield should converge back to the initial source, just as for acoustic waves as seen in the previous part.

The theory of such an electromagnetic time reversal cavity has been done recently. When considering electromagnetic waves, the Green's function have to be replaced by dyadic Green's functions. For a narrowband signal of oscillating pulsation $\omega$, the time reversal focusing on position $r_{0}$ is equivalent to phase conjugation, and it can be demonstrated starting from Lorentz reciprocity theorem that the electric field generated at position $r$ simply writes $[32,33]$

$$
E_{\mathrm{RT}}(r, \omega)=-2 i \mu_{0} \omega^{2} \operatorname{Im}\left(\overleftrightarrow{G}\left(r_{0}, r, \omega\right)\right) \cdot \mathbf{P}^{*},
$$

where $\mu_{0}$ is the permeability of vacuum, the tensor $\overleftrightarrow{\mathbf{G}}$ stands for the dyadic green function of the medium, and $\mathbf{P}$ represents the initial vector dipole source. When the dyadic green function is the free space one, it results in a cardinal sine function: the focal spot is diffraction limited to $\lambda / 2$, as we will experimentally prove it. When the time reversal mirror is placed in the near field of the initial source, the green function takes into account the evanescent component of the field generated by the source and a smaller spot can be obtained [34]. Note that the field amplitude at the focal point is proportional to the imaginary part of the Green's function which is itself proportional to the so-called local density of states [35] (LDOS).

Again, considering that time reversal uses broadband excitation, the resulting field takes advantage of the frequency diversity. For an excitation with a flat bandwidth, all frequencies add up in phase at a given time, the collapse time $(t=0)$. At this specific time, the time reversed field writes [23]

$$
E_{\mathrm{RT}}(r, t=0) \propto \mu_{0} \omega^{2} \int_{\text {Bandwidth }} \operatorname{Im}\left(\overleftrightarrow{G}\left(r_{0}, r, \omega\right)\right) \cdot \mathbf{P}^{*} d \omega .
$$


At this stage, two remarks can be done. The first one is that compared to scalar waves, polarized wave offer more degrees of freedom using TR focusing since the polarization is conserved in a TR experiment. This can be of interest when trying to focus energy or in wireless communications issues, for instance, for multiple-input multiple-output communications (MIMO). The second remark that one can notice is that for electromagnetic waves as well, the TR field created at the collapse time in a medium, where a modal analysis can be done, can simply be understood as a sum of all the modes within the bandwidth interfering coherently at the original source position. This will have great implications in the next parts of the paper, when we will consider subwavelength scaled medium for TR focusing beyond the diffraction limit. Before that, we will show some experiments of TR focusing in homogeneous media or in other words, diffraction limited.

Again, it would be hardly possible to design an electromagnetic time reversal cavity and the number of antennas to be used for a TR experiment has to be reasonable. In order to demonstrate the first results of TR with electromagnetic waves, we have therefore used the knowledge acquired thanks to the experiments realized in the ultrasound domain. But another problem occurs while translating TR from the field of acoustic towards that of electromagnetic waves. Indeed, electromagnetic waves that can be easily manipulated in laboratories (e.g., is the range of the centimetres) have much higher frequencies than ultrasound. One could think that achieving TR at such high frequencies is not possible. In fact, we have proved that TR at any frequency can be realized using modulated signals, by simply time reversing the complex envelope of the latter and phase-conjugating the carrier [10]. Using IQ modulated radio-frequency signals in a reverberating chamber, similar to previous experiments in ultrasound, we have demonstrated spatial focusing of microwave using TR in [11]. Those experiments were done at the wifi frequency of $2.45 \mathrm{GHz}$, using a bandwidth of about $100 \mathrm{MHz}$, and in a homemade $1 \mathrm{~m}^{3}$ reverberating chamber (Figure 3(a)). The focal spot obtained by TR, measured as the temporal maximum of the absolute value of the field received on an array of 8 monopoles $\lambda / 8$ spaced, is presented in Figure 3(b).

As expected, the focal spot obtained is about $\lambda / 2$ wide, the wavelength being defined at $2.5 \mathrm{GHz}$, since it is the integration over a rather narrow bandwidth of a sinc function. Quite interestingly for many applications, although the TR operations are done at low frequencies below $100 \mathrm{MHz}$, the waves inside the cavity are focused at the carrying frequency. This means that provided that modulators be available, it is possible to focus waves on any given diffraction limited spot by increasing the frequency of the carrier, and without changing the electronics devoted to the TR operation. This has interested researchers in the domains of microwave medical imaging and therapy [20] as well as in sensing and imaging [12-15]. But a very important question that remains is as follows can the focal spot obtained by TR be independent of the wavelength at which TR is achieved? Rephrased at the light of (4), this question cuts down to: can we find medium which have dyadic Green's functions that possess spatial variations much smaller than the wavelength in free space? In the next part, we will show that a positive answer to this question has been experimentally given.

\section{Far Field Subwavelength Focusing Using Time Reversal in an Array of Resonant Wires}

Having the ability to focus waves on dimensions much smaller than the wavelength from the far-field, that is, while controlling the field at the scale of the wavelength, has always been a major field of study. Indeed, such a concept has many potential applications in various fields such as sensing, lithography, wireless communications using MIMO compact antennas, or more generally any domain where energy has to be deposited onto narrow focal spots. There exists, however, a very solid barrier that prevents waves from being focused on a subwavelength scale, in homogeneous media. The latter, known as the diffraction barrier or the Abbe's limit, can be explained physically in rather simple words.

A source field placed in a given medium, whether extended or point-like, possesses a spatial profile that can vary much faster than the wavelength at which it radiates. This can be the case for instance of a small dipole, whose near field contains fast varying components that decay as the distance cubed, or for a rough surface illuminated by a plane wave, which scatters the field into many wavevectors some of them being much larger than in the host medium. If one wants to study the propagation of such a wavefield in a given direction, the easiest way to do it is to perform a Fourier transform of this field on a plane perpendicular (transverse) to the considered propagation direction. The spatial components of the source field which vary faster than the wavelength have, in this transverse plane, wavevectors whose norm exceeds the wavenumber in the medium. Since the norm of any wavevector must equal the wavenumber, those subwavelength spatial variations must present a purely imaginary wavevector in the direction of propagation. Those waves are called evanescent ones, and due to their imaginary propagation constant, they decay exponentially from the source. This explains why from the far-field it seems impossible to focus onto subwavelength spots: all the waves that carry spatial information smaller than the wavelength are lost during propagation. Goodman describes this diffraction limit in other words; he characterizes the propagation from one plane to another as a low pass filter whose cutoff wavevector is that of the waves in the medium [5].

One way though, in order to circumvent this diffraction limit, is to make use of so-called near field techniques. The idea of near field imaging dates back to the beginning of the 20th century with a proposal that made Synge during an epistolatory conversation with Einstein about ways to beat Abbe's limit [36]. The proposed method consists in illuminating a very small portion of an object using an opaque screen perforated with a deep subwavelength hole. The point-like source is then scattered by the object into a wave than radiates in free space, hence carrying local and subwavelength information about the object. From this 


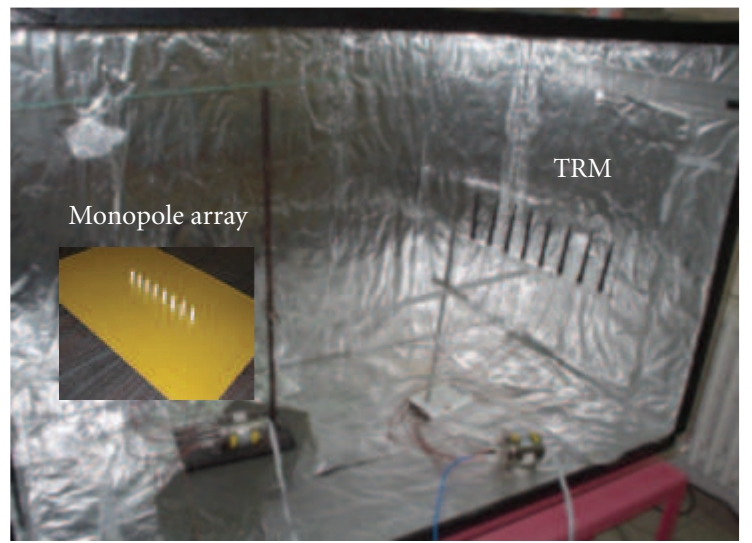

(a)

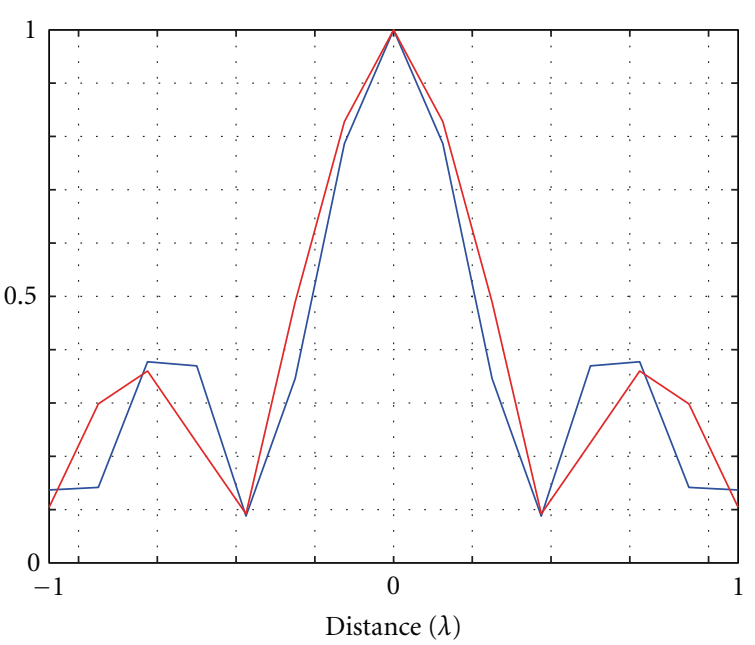

(b)

FIgure 3: (a) Picture of the experimental setup. The experiment is realized in a $1 \mathrm{~m}^{3}$ homemade reverberant chamber. The TRM consists of 8 commercial antennas $\lambda / 2$ spaced, while the field is measured at the TR focal spot using an array of 8 monopoles on a ground plane $\lambda / 8$ spaced. (b) Typical focal spot obtained using TR (Blue experimental, Red Analytical taking coupling into account). The focal spot in an homogeneous medium is diffraction limited to $\lambda / 2$, which reduces here to $0.4 \lambda$ due to the coupling between the monopoles.

proposal, the concept has been declined in many different ways and has led to the development of near field scanning optical microscope, that is based on aperture or apertureless probes, and whose principles are sensibly identical [37-40]. Basically the probe, for instance, the elongated tip of an optical fiber, is brought in the very near field of the object to be imaged. This very subwavelength apex scatters locally the evanescent field that is present at the surface of the object to be imaged, some of it being scattered as propagating waves. A conventional imaging system collects the light radiated by the probe, and the information of the object's near field at the specific position of the tip is then extracted. Scanning the tip over the object point by point allows one to reconstruct the near field pattern of the object, and resolutions well below the diffraction limit have been reached using such microscopes.

Such method, however, appears quite limited from a focusing point of view. Indeed while dealing with focusing issues, the aim is to be able to focus a wave on several points without the need of any mechanical moving stage. The tip used in near field scanning methods being punctual, focusing onto such an object would be quite useless since it would not authorize position controllable focusing without a mechanical part, not mentioning the issue of multiple foci. Nevertheless, this method has inspired us since it makes use of the fascinating concept of evanescent to propagating waves conversion that is necessary in order to focus waves on a subwavelength scale and from the far field. The concept that we have imagined is the following. Suppose that we construct a medium that is able to convert the evanescent field created by a source into propagating waves that can be recorder in the far field. Then, using TR from the far field, because the wave equations are reversible, we should be able to focus back the wave on its initial position, and since the evanescent to propagating wave conversion is reciprocal [41], we should be able to obtain a subwavelength resolved focal spot. If we place a bunch of those sources at distance much smaller than the wavelength in the fabricated medium and learn the impulse response from those sources to a TRM, then we should be able to focus independently on each of them, hence demonstrating that the diffraction limit is beaten.

This is exactly the idea we have exploited in [22] in order to create focal spots much thinner than the wavelength. Indeed, using time reversal in a random collection of metallic scatterers, we have demonstrated time reversal focusing of microwaves from the far field onto spots as thin as $1 / 30$ th of a wavelength in air.

One point that was not obvious in the experiments of [22], however, was that the wires were all approximately of equal length. Considering this microstructured medium through this new prism, they can be visualized as an array of resonant wires, since they are all around a quarter wavelength long, and placed on top of a copper ground plane (i.e., resonant monopole). Such an array of resonators should behave as a matrix of coupled oscillators, similar to a system of $N$ coupled mass/spring systems. This is the idea that we have explored in order to grasp the physics of such a medium and infer the links with the results of TR focusing on a subwavelength scale.

In order to study far field TR in such an array of resonant unit cells, we have simplified the system; the medium (Figure $4(\mathrm{a})$ ) is composed of a periodic array of $N=20 * 20$ equal length copper wires (length $L=40 \mathrm{~cm}$, period $a=$ $1.2 \mathrm{~cm}$, wire diameter $d=3 \mathrm{~mm}$ ). The wires are oriented along the $z$ direction and periodically spaced in the $(x, y)$ plane. Furthermore, we perform all our measurements and simulations in free space or in an anechoic chamber in order to decouple the effect of the medium and those of the reverberating chamber used in [22]. Leaving the random 


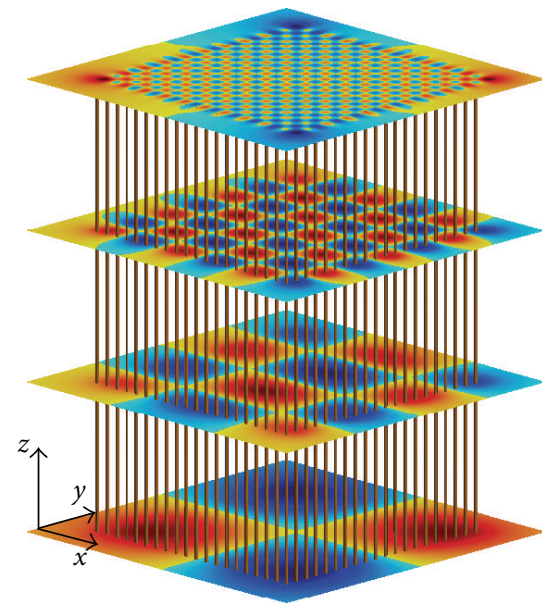

(a)

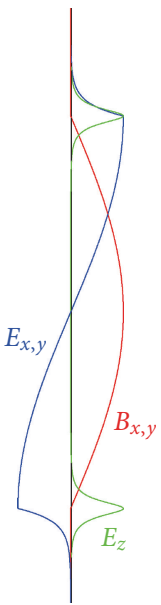

(b)

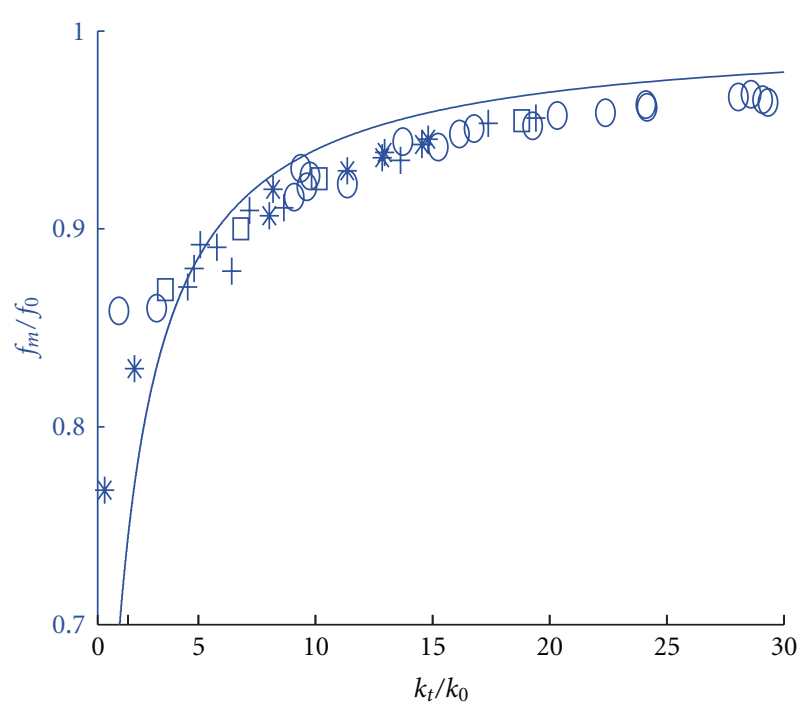

(c)

Figure 4: (a) The experimentally and numerically studied resonant wire array. The $20 * 20$ wires are $1.2 \mathrm{~cm}$ periodically spaced on a square grid and are $3 \mathrm{~mm}$ in diameter and $40 \mathrm{~cm}$ long. Superimposed are mapped the electric field of 4 Bloch modes out of the 400 that are supported by the structure. (b) Shape of the TEM field inside the structure. The transverse magnetic (electric) field is maximum (null) in the middle of the medium of the structure and null (maximum) at the interfaces with air. Only an electric evanescent component exists outside of the medium in the $z$ direction. (c) Dispersion relation linking the transverse wavevectors $k_{t}$ (normalized to $\pi / a$ ) of the modes to their resonant frequencies $f_{m}$ (normalized to the resonant frequency of a single wire $f_{0}$ ). The solid curve is analytical (equation (5)), while the symbols represent various modes obtained numerically. This curve shows that the Bloch modes which are evanescent in the $z$ direction (the curve is under the light line) show a dispersive behaviour.

medium for a periodic array of identical resonators presents two major advantages: first, a Bloch mode analysis can be performed, which greatly simplifies the study, and second, the medium can be analysed within the frame of the wire media that has been deeply studied in the past for different purposes $[40,41]$.

We have realized an in-depth study of this array of wires, and we will give the principal results in order to explain the principles of TR in such a medium. Part of this study has been published in [42]. We mention first that obviously, since this system is analogous to an array of coupled mass/spring systems, we expect that the system can be described in terms of eigenmodes and eigenfrequencies. The latter appear due to the strong coupling between the wires since they are very densely packed. Indeed, the first resonance $f_{0}$ of a wire is around $375 \mathrm{MHz}$, and they are therefore organized on a very deep subwavelength at the equivalent resonant wavelength $\lambda_{0}=80 \mathrm{~cm}$, that is, a period equals $\lambda / 70$. The very difference between such a medium and a wavelength scaled one, for example, a classical photonic crystal, is that in our case the Bloch modes can be very subwavelength. Indeed, in such a system, Bloch theorem imposes that we find $N$ modes if we have $N$ oscillators. Those modes possess wavevectors $k_{t}$ in the $(x, y)$ plane (which we will thereafter refer to as the transverse plane) linearly distributed onto the interval $[0, \pi / a]$. Hence, the spatial variation of the modes supported by the deep subwavelength array of resonant wires ranges from the transverse size of the system for the fundamental mode toward the period of the system for the higher mode. Here we start to understand a very important feature of the system used in [22] and studied deeper in [42]: such a medium supports deep subwavelength modes that will be able to couple to the evanescent components created by a source placed either inside or in the very near field of the medium. Lets us now look at the dispersion relation of such modes or equivalently the relation between their eigenvectors and eigenfrequencies.

Such a system of $N$ periodic resonators should be analysable within the frame of classical coupled oscillators: a set of $N$ equations is written modelling the behaviour of the current in each wire, and including a coupling constant linking this specific wire to its neighbours. This approach, however, requires the knowledge of the coupling matrix, some computations, and there is actually a much more elegant way to obtain the desired results. The idea of this analysis is to start from an infinite wire medium of same periodicity and wire diameter as the one utilized here. Such medium, known as the wire medium, supports only transverse electromagnetic modes (TEM modes similar to those propagating in coaxial lines), since TE and TM modes turn out to be evanescent due to the very subwavelength period [43, 44]. Those TEM modes are known to be dispersionless: their longitudinal wavevectors $k_{z}$ are independent of the transverse one and equal $\omega / c$, where $\omega$ is the pulsation of the wave and $c$ its celerity in the host medium. When a set of TEM modes, each one presenting 
a complex phase (the decomposition of a point source for instance) propagates inside this medium, it remains unchanged independently of the distance of propagation in the $z$ direction, which according to some authors explains the phenomenon called canalization [43, 44].

If we start by cutting the wires along the $z$ direction such that they now present a finite length $L$, placing the wire medium between the $z=-L / 2$ and $z=L / 2$ planes, things become quite different. Indeed, the dispersionless TEM modes are trapped inside the medium and they propagate back and forth along the $z$ direction: they experience a Fabry-Perot type of cavity. Interestingly, the properties of this Fabry-Perot cavity depend on the transverse wavevector of the modes $k_{t}$, which in turns induces dispersion. Very naïvely, one can understand this in the following manner; the TEM modes are all evanescent outside the medium in the $z$ direction at the $z= \pm L / 2$ interfaces (Figure 4(b)) since they are subwavelength, or equivalently, since their resonant frequencies lie below the light line. It is well known that when a wave impinges from a medium 1 onto an interface with a medium 2 and gives rise in the latter to an evanescent wave, it adds a phase term to the reflection coefficient at the interface. This effect, responsible notably of the Goos and Hänchen shift [45], depends on the penetration depth of the evanescent wave inside medium 2: the longer this penetration depth, the larger the added phase. Taking this effect into account, it is pretty easy to obtain a relation between the TEM modes transverse wavevector $k_{t}$ and their eigenfrequencies:

$$
\tan \left(\frac{k L}{2}\right)=\frac{\sqrt{k_{t}^{2}-k^{2}}}{k}
$$

where $k$ denotes the host medium wavenumber, and hence the frequency (in our case the host medium is air and $k=$ $\left.k_{0}=\omega / c\right)$.

This dispersion relation is plotted in Figure 4(c) alongside simulation results obtained from CST Microwave Studio. From now on, it is clear that the TEM modes are no longer dispersionless inside the medium, which will have many implications. Indeed, if one uses an electrically small source in the near field of the medium at the $z= \pm L / 2$ interfaces, the field created by the latter decomposes onto the eigenmodes of the system. Since the modes are dispersive, namely, each mode possesses a different resonant frequency, and if the source is broadband and covers all the dispersion relation, then the Fourier decomposition of the source onto the modes is transposed into the spectrum of the field inside the structure. This means that the spatial information of the source on a subwavelength scale down to the period of the structure can be converted into a temporal waveform or a complex spectrum thanks to the dispersion that occurs inside this subwavelength scaled resonant medium. This aspect constitutes the first out of the two fundamental concepts that permit beat the diffraction limit using time reversal focusing from the far field.

We have shown that a subwavelength source placed in the near field of one interface of the finite length wire medium can decompose onto the subwavelength modes of the system provided that it is broadband and can excite all of the resonant frequencies of the modes. But up to now, the medium still remains infinite in the $(x, y)$ plane. Cutting the system in the $(x, y)$ plane in order to obtain the finite size medium that we have studied has majors consequences. First of all, evidently, when we restrict the medium to $N=20 * 20$ wires, the dispersion relation is no longer continuous, but the transverse wavenumbers $k_{t}$ become discrete on the interval $[0, \pi / a]$ since we have only $N$ modes. But there exist a much more important outcome of the finite transverse dimensions of the structure: the modes become leaky in free space due to finite size effects. Of course, the efficiency of the conversion from those subwavelength modes to free space radiations is quite poor, and the more subwavelength the modes, the less efficient the conversion.

What seems to be very limiting though is actually not a problem but rather a solution. Indeed, since the array of wires is resonant, energy can be built inside the structure with time. This implies that the modes which cannot exit the structure easily, the most subwavelength ones, are trapped inside the medium during a much longer time. Hence, they present a much higher quality factor, and on a monochromatic point of view their amplitude inside the medium is much higher than that of the source, due to a resonant amplification, or equivalently the Purcell effect [46]. From energy conservation considerations, one can easily infer that if the materials are lossless and even though the conversion of the modes to free space radiation is very weak, due to this resonant effect all the modes radiate equally efficiently in free space! This represents the second fundamental of such finite size subwavelength-scaled resonant media: the subwavelength modes very low conversion to free space radiations is totally counterbalanced by the resonant enhancement of the modes. This explains why those modes actually radiate efficiently in free space, even though they are deeply subwavelength inside the medium (down to the period of the medium).

Together with the first principle, the physical mechanism of our concept can be summarized as follows. First, a source placed in the near field of our medium emits a broadband field that decomposes onto the eigenmodes of the latter. Due to dispersion, the spatial decomposition is converted into a temporal/frequency signature, which resonates inside the medium, with the quality factor of the resonance being higher for the most subwavelength modes. Then, due to the finite size effects and the resonant enhancement of the modes, each mode radiates with an equal efficiency in free space. This explains the efficient conversion of the subwavelength information into free space radiations of [22], and the fact that TR can give subwavelength resolution in this medium; indeed, if the conversion is possible from the source to free space, then the time reversed one is possible due to the reversibility.

We have verified experimentally and numerically those principles. Numerical simulations using lossless metal have shown us that using a $20 * 20$ wire medium, all the spatial information of a source is radiated in the far field with equal efficiency, regardless of its subwavelength nature [42]. We have also repeated the TR experiments of [22] using 

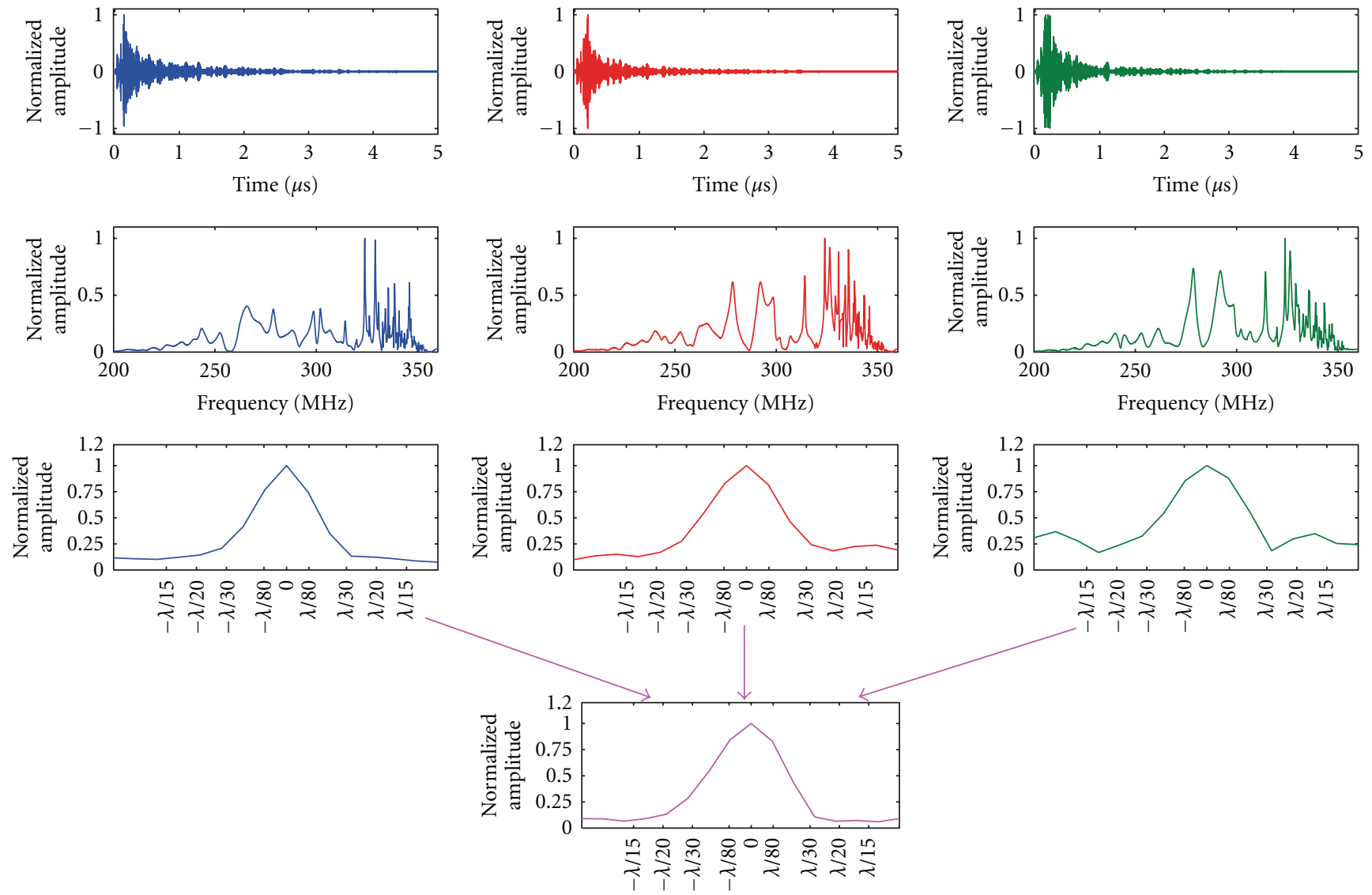

FIGURE 5: Experimental results in an anechoic chamber of temporal waveforms (first row), frequency spectrum (second row), and focal spots obtained by TR (third row), for the 3 antennas placed in the far field of the structure at relative angles $0^{\circ}, 45^{\circ}$, and $90^{\circ}$. In each case, the signals are very elongated due to the resonant nature of the medium only, the spectrum displays increasing quality factors for frequencies approaching $f_{0}$, and the focal spots are $\lambda / 25$ wide. The last row presents a focal spot obtained using TR with the 3 antennas at a time. The focal spot is again $\lambda / 25$ wide and the sidelobes are lowered.

our periodic system in an anechoic chamber. As can been seen in Figure 5, we have recorded the field produced by a small monopole placed in the near field of the medium using 3 antennas in the far field of the structure. Those three antennas are placed at $0,45^{\circ}$, and $90^{\circ}$ around the medium, and we record the impulse response between these antennas and the monopole placed in the near field of the medium. The emitted signal is a $10 \mathrm{~ns}$ pulse centred at $300 \mathrm{MHz}$. It is clear from the temporal signals that even though placed in an anechoic chamber, the impulse response between the monopole and the three antennas is very long $(1.5 \mu \mathrm{s} \mathrm{rms}$, i.e., 150 times the original pulse). This means that the array of resonant wire itself constitutes a very high $Q$ cavity for the TEM modes that resonate inside it. Next, considering the spectrum of the recorded impulse responses, it is clear that our analysis is correct, since we can see that the modes that are closer from the resonant frequency of a single wire (375 MHz) show increasing quality factors.

We have also performed TR focusing using the three far field antennas. To that aim, we first emit with the small monopole a $10 \mathrm{~ns}$ short pulse centred around $300 \mathrm{MHz}$. We then measure with a high speed digital scope the impulse responses between this monopole and each of the three far field antennas placed in the anechoic chamber. Those signals are then time reversed, that is, flipped in time. We generate the corresponding waveforms with a high sampling rate arbitrary waveform generator and send back those signals by the far field antennas acting now as emitters. Measuring the field received on an array of monopoles placed around the initial source position in the near field of the wire medium, we have access to the temporal signal obtained after TR. We define the focal spot obtained after time reversal as the maximum over time of the modulus of the amplitude received on each monopole. This operation is first realized with one of the three far field antennas at a time, and then with the three of them simultaneously. Clearly, TR focusing gives a focal spot that is deeply subwavelength (around $\lambda / 25$ ) using any of the three far field antennas, that is, using a single antenna and in free space (anechoic chamber). As one should expect, when the three antennas emit simultaneously the time reversed signals, we obtain a similar focal spot, but the amplitude of the signal outside of the focal spot, which we call sidelobes, is lower owing to the utilization of multiple antennas. 
A brief letter of this detailed study can be found in [42] and the rest of it will be published elsewhere. In [42], we call such a system a resonant metalens since it can project the image of an object with subwavelength details in the far field, coded in a temporal/frequency signature. We prove in those papers both TR focusing under the diffraction limit and imaging from the far field with subwavelength resolution based on the same concepts. Of course, here we did not mention the effect of material losses. Evidently, losses will diminish the quality factors of the most subwavelength modes propagating in the structure, which in turn will reduce their resonant enhancement and finally their radiation efficiency. This, in turns, will set a limit to the resolution of the system, whether it is used for TR focusing under the diffraction limit or for purposes of subwavelength imaging.

Finally, it is worth making a link between the results obtained here and the formula (4) linking the result of TR at the collapse time and the dyadic Green's functions of the medium. Indeed, in such a periodic system, the Green's functions of the medium are the modes that exist in the latter at each frequency. Equation (4) states that the result of TR focusing at the collapse time is the integral of the dyadic Green's functions projected on the dipole vector of the initial source. Very concretely, if one's aim is to focus using TR on a deep subwavelength scale, the idea is to sum a lot of independent subwavelength modes in phase at the focal spot and the collapse time. This is exactly what TR does, since it compensates the phase of the modes excited in the structure from the far field, such that they focus on the initial source position at a definite time. From this approach, it is clear that such results can be obtained provided that we are able to construct a medium supporting subwavelength modes, which must be independent (that is, the medium must be dispersive) and must present ohmic losses as low as possible. We will show in the next part that resonant wires, even though they are very convenient for this purpose due to their small transverse dimensions, are not the only candidates.

\section{Using Any Subwavelength Resonator to Achieve Far Field TR Focusing below the Diffraction Limit}

From the results of the last part, it appears that any medium of finite size supporting subwavelength modes, consisting of resonant unit cells and displaying dispersion can be utilized for the sake of subwavelength focusing from the far field using TR. Very conveniently, many of such media have been studied recently in the field of metamaterials. Basically, metamaterials are engineered materials made out of a periodic array of unit cells which exhibit interesting properties that can be tailored [47]. Most metamaterials are designed with a unit cell that is resonant and of subwavelength size. Examples of such unit cells include Split-Ring resonators (SRR), parallel cut wires, complementary SRR, and so forth. Usually such materials are analysed within the frame of the effective medium theory, and effective parameters are obtained through an averaging of the field across one unit cell. Materials with negative permeabilities and permittivities can be obtained, those possessing a band with double negative parameters falling into the class of negative index media [48].

In our case, we are not interested in averaging procedures in order to extract effective parameters since our focus is on finite size media. Such finite size media necessarily support modes which can be calculated using Bloch formalism. Provided that the size of its unit cell be subwavelength and resonant, a medium should behave just as the medium made out of resonant wires that we have studied before. Indeed, the modes supported by the medium for the first band should be evanescent since their dispersion relation, analogically to polaritons, lies under the light line. Hence, such modes should be once again trapped inside the medium which then acts as a cavity for those trapped modes. Under the condition that the unit cells be coupled, which should be the general case since arranged in a dense array subwavelength resonators will strongly interact with each others, the medium should also have a dispersive behaviour. This should permit to transpose the subwavelength spatial information of a source into the temporal/frequency signature of the field inside the medium. Finally, because we consider only finite size media, finite size effects should also ensure a conversion from the subwavelength modes to free space radiation whose weak efficiency should be compensated by the resonant enhancement of the modes in the medium.

Therefore, we argue that if one designs a resonator of subwavelength size and utilizes it in order to construct an array of such resonators, the latter being closely spaced, focusing using TR should produce subwavelength spots in such media. The focal spot size will in the best case be limited by the period of the medium, but may be limited to larger sizes depending on the losses of the system. We have verified our assertion with various unit cells such as high index dielectric cubes, spiral resonators, loaded wires, and Split-Ring resonators. The results obtained totally agree with our statement, and in all cases, at least numerically, TR focusing resulted in subwavelength focal spots. Here, for the sake of space limitations, we will only present the case of an array of Split-Ring resonators.

The unit cell of the structure, schematised in Figure 6(a), consists of four split rings. A first $8 \mathrm{~mm}$ wide SR is inserted inside a $1 \mathrm{~cm}$ wide one, the gaps being tilted of $180^{\circ}$. The copper strips are $0.5 \mathrm{~mm}$ wide and the gaps $1 \mathrm{~mm}$ wide. A structure identical to the latter is placed in front of the first one, tilted $90^{\circ}$ from the first one. Each of those structures are printed using copper on the two sides of a $1 \mathrm{~mm}$ thick low loss dielectric substrate (Duroïd 500). The structure is designed in that way for two purposes. First, there are 4 SR such that the resonant frequency of the cell is lowered enough in order for the equivalent resonant wavelength to be much larger than the unit cell width. The second aim is to simplify the analysis of the following experiments by keeping only the magnetic resonance of the cell. Indeed a Split-ring usually possesses two fundamental resonances, one magnetic and the other electrical, both of which can be excited depending on the polarization of the field. Because on each side of 


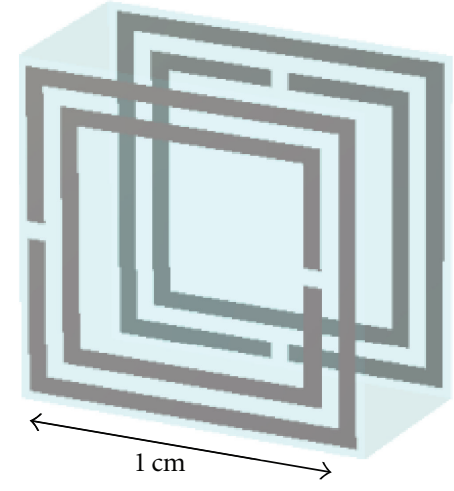

(a)

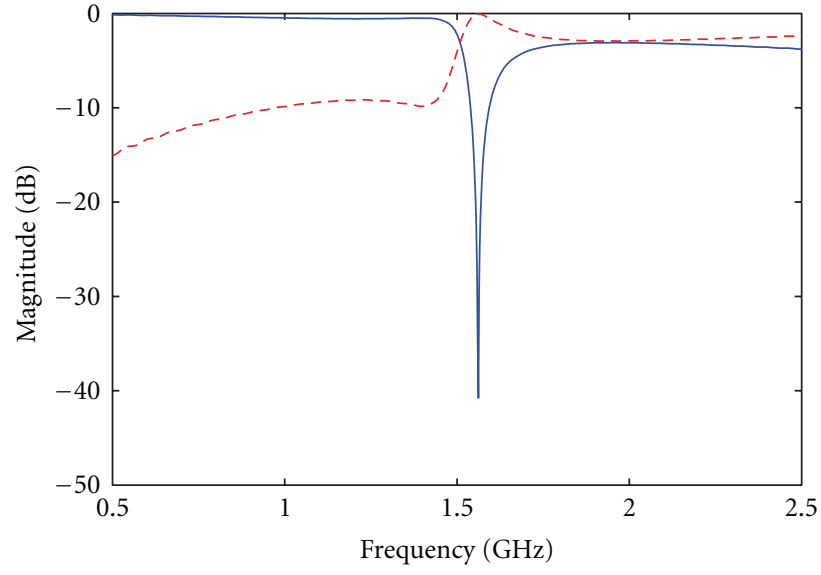

(b)

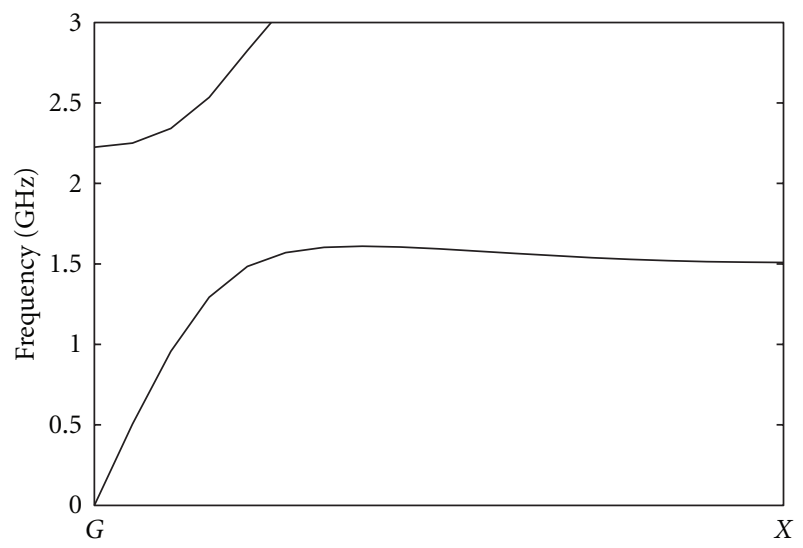

(c)

FIGURE 6: (a) Unit cell of the SRR used in our numerical simulations and experiments. 4 basic SRR are used to design this cell in order to suppress the electric resonance. The SRR parameters are given in the text. (b) S parameters of a single cell obtained numerically using CST Microwave Studio. The $S 12$ (transmission, solid blue curve) shows a sharp deep around $1.6 \mathrm{GHz}$ while the $S 11$ (return loss, dashed red curve) has a maximum at $1.6 \mathrm{GHz}$. Both curves indicated a sharp resonance of the SRR at 1.6 GHz. (c) Band structure of a plane of such SRRs arranged a square array of period $1.1 \mathrm{~cm}$. The lower branch is the one that coupled evanescently to our source. Those modes are evanescent perpendicularly to the array since the band lies under the light line. The upper branch shows leaky modes (with good coupling to free space radiations and limited $k_{t}$ ) that are not interesting for our concept. Clearly the interesting subwavelength branch is dispersive which is crucial for our concept.

the substrate the cell possesses gaps tilted $180^{\circ}$ one from the other, the electric effects cancel and solely the magnetic resonance of the cell survives.

We have studied numerically the structure using CST Microwave Studio in order to obtain the $S$ parameters of a single unit, that is, the transmitted and reflected fields for a wave impinging on such a unit cell. They are plotted in Figure 6(b) as a function of the frequency. A clear resonance appears in the signal around the frequency $f_{0}=1.7 \mathrm{GHz}$. Given the size of the unit cell, $1 \mathrm{~cm}$, and its equivalent resonance wavelength which roughly equals $18 \mathrm{~cm}$, this resonator can be described as deeply subwavelength. We have then numerically studied the behaviour of a planar array of such resonators, arranged on a square lattice of period $1.1 \mathrm{~cm}$. Again, CST Microwave Studio has been used using the mode solver and we have obtained the dispersion relation of an infinite plane of such unit cells. This dispersion relation is plotted in Figure 6(c). Due to the coupling between the SR, this planar medium is dispersive, as it was demonstrated before [49]. This way, it is clear that if we put a small loop antenna in the near field of this planar array of SR and excite the loop with a broadband signal, the spatial information of the loop will be transferred to the spectrum of the field propagating inside the 2 -dimensional medium. Fabricating a finite size version of such a medium should then permit us to focus waves on a deep subwavelength scale in this medium using far field TR.

We have fabricated such a structure using an array of $10 * 10$ unit cells respecting the aforementioned parameters. This array can be seen on the photo of the experimental setup presented in Figure 7(a). In our experimental demonstration, a small loop antenna oriented in the same direction as the SR is placed in the near field of the array of SR, at about $2 \mathrm{~mm}$ from the surface of the sample. This loop 


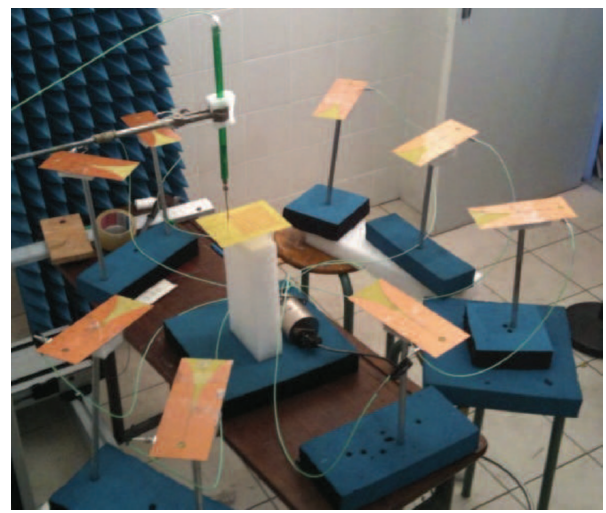

(a)

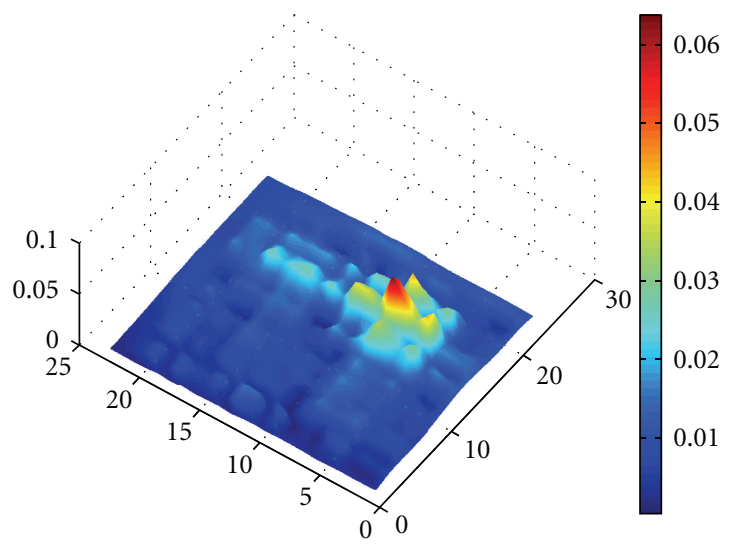

(b)

Figure 7: (a) Photo of the experimental setup. The array of $10 * 10$ SRR is printed onto Duroïd 500 which has a low loss tangent. A small loop (attached on a grey tube) is used as the near field probe to measure the impulse responses from the 8 Vivaldis antennas to every SRR of the array. This probe can be translated in the $x, y$, or $z$ directions. A multiplexer (grey cylinder) is used to switch between the Vivaldi antennas. It is linked to the first port of a network analyser, while the second is connected to the probe. (b) Field at the collapse time after TR calculated using the set of impulse response for a passive TR focusing on the SRR $(3,7)$, two units stand for one period of the array. Clearly, the focal spot is one period wide, that is, $\lambda / 18$, deeply subwavelength.

antenna is attached to a $3 \mathrm{D}$ positioning system that can be utilized to scan the magnetic field on top of the sample. This loop antenna is connected to one port of a network analyser. The other port of the network analyser is connected to an electromechanical switch which permits to switch between 8 Vivaldi antennas that are placed around the array of SR, at about 2 wavelengths in order to avoid any near field coupling, and which span over $360^{\circ}$, approximately every $45^{\circ}$. Those planar Vivaldi antennas are oriented parallel to the planar array of SR, meaning that the electric field is polarized parallel to the plane of SR as well. This way, the magnetic field produced by those antennas is perpendicular to the array of SR and can excite the modes of the medium.

We have performed a virtual TR focusing experiment using the following procedure. A set of impulse responses between the 8 Vivaldi antennas and the loop antenna is acquired when this small probe is placed on top of one of the hundred of SRs. The operation is repeated for every position of the loop antenna on top of the array, that is to say, we acquire the set of responses between the 100 SRs and the 8 Vivaldi antennas representing the time reversal mirror. This bank of impulse response can be written $h(V, i, j, t)$ where $V$ denotes the index of the TRM antenna between 1 and 8, and $(i, j)$ stands for the couple of indices of the SR on the array. This signal bank is then utilized in order to compute the result of a time reversal focusing experiment on any of the 100 SRs. If we want to emulate the results of a TR focusing on the SR of indices $(i, j)$ on the array and from the 8 antennas of the TRM, we can compute the maximum over time of the fields that could be measured on top of every SR of the array when the TRM sends the impulse responses of $\operatorname{SR}(i, j)$. This focal spot estimation writes

$$
S(k, l)=\max _{\text {time }}\left(\sum_{V=1}^{8} h(V, i, j,-t) \otimes h(V, k, l, t)\right)
$$

which can be rewritten simply in the Fourier domain in order to accelerate the computation as

$$
S(k, l)=\sum_{V=1}^{8} \int_{\text {Bandwidth }} H^{*}(V, i, j, \omega) H(V, k, l, \omega) d \omega .
$$

The result of such a focusing experiment has been obtained using the experimentally measured impulse responses, and we map it in Figure 7(b). Clearly, the size of the focal spot is limited by the period of the array to $\lambda / 18$ at the central frequency of the original emitted bandwidth. This means that the losses on the substrate and the copper do not hamper the TR focusing, owing to the small loss tangent of the Duroïd. As a sidenote, we would like to mention that other focusing techniques may give better spatial focal spots depending on the losses experienced by the modes in the structure. Indeed, TR is a matched filter, meaning that it maximizes the energy focused at the focal spot and the collapse time [50]. An inverse filter may give better spatial results if the medium is too lossy by increasing the emitted energy in the most subwavelength modes, at the price of a reduced efficiency [50-52].

\section{Conclusion}

The goal of this was to introduce TR as a method for focusing below the diffraction limit from the far field in designed subwavelength scaled and resonant media. To that aim, we have introduced the basic principles of TR through a brief review of some results obtained using acoustic waves. We have shown that a TR focal spot can be explained in terms of a sum over the bandwidth of the Green's functions of the medium, which happen to be proportional to the modes of the medium if the latter can be analysed within 
a modal approach. Starting from those original results, we have underlined that they can be transposed to the case of electromagnetic waves. More specifically, we have shown that in terms of focal spot TR behaves exactly identically with electromagnetic waves than it does in acoustics, notwithstanding the polarized nature of the latter which must be accounted for through the use of Dyadic Green's functions. We have also reminded the reader how the drawbacks linked to the high frequency character of microwaves can be circumvented through the use of modulation techniques. This has led us to introduce our first experimental proof of concept of subwavelength focusing using far field TR in a random collection of metallic scatterers. This experiment has been explained in terms of conversion of evanescent waves to propagating ones, and linked to the reciprocity of evanescent waves. Then we have given evidence that this initial demonstration could be analysed within the simplified model of a subwavelength periodic array of resonant wires. The main characteristics of such a medium have been presented: dispersion allows the evanescent components of a source to couple to the subwavelength Bloch modes supported by the structure, while the weak conversion to propagating waves of the latter is counterbalanced by the resonant enhancement of the modes. We have verified that $\mathrm{TR}$ in such media indeed provides deep subwavelength focal spots whose widths are limited by the losses of the structure to $\lambda / 25$ in our experimental demonstration. At the light of this new approach, we have generalized the concept and argued that any dense and finite-size array of subwavelength resonator constitutes a medium which can be utilized to break the diffraction barrier using TR focusing. This has been evidenced using an array of Split-Ring resonators onto which focal spots as thin as $\lambda / 18$, the period of the array, have been measured.

We believe that this approach because of its generality and simplicity will have very interesting applications in various fields such as medical therapy, sensing, or telecommunications. We would like to underline that this concept is far from being limited to the domain of microwaves: we are currently working on its transposition to the optical domain using silver nanowires and we have already published a study with audible sound using arrays of Helmholtz resonators, that is, soda cans [53]. This broadens the range of the envisioned applications and testifies of the generality of this principle. Finally, we would like to add that such media, which we have called "resonant metalens" for their capability to project on the far field the near field information of a source regardless of its spatial extension, can also be utilized as imaging systems with subwavelength resolution.

\section{References}

[1] M. Fink, “Time reversed acoustics," Physics Today, vol. 50, no. 3, pp. 34-40, 1997.
[2] A. Derode, P. Roux, and M. Fink, "Robust acoustic time reversal with high-order multiple scattering," Physical Review Letters, vol. 75, no. 23, pp. 4206-4209, 1995.

[3] A. Derode, A. Tourin, and M. Fink, "Random multiple scattering of ultrasound. II. Is time reversal a self-averaging process?" Physical Review E, vol. 64, no. 3, pp. 366061-366113, 2001.

[4] A. Derode, A. Tourin, J. de Rosny, M. Tanter, S. Yon, and M. Fink, "Taking advantage of multiple scattering to communicate with time-reversal antennas," Physical Review Letters, vol. 90, no. 1, Article ID 014301, 4 pages, 2003.

[5] J. Goodman, Introduction to Fourier Optics, Roberts \& Company Publishers, Greenwood Village, Colo, USA, 2005.

[6] C. Draeger and M. Fink, "One-channel time reversal of elastic waves in a chaotic 2D-silicon cavity," Physical Review Letters, vol. 79, no. 3, pp. 407-410, 1997.

[7] W. A. Kuperman, W. S. Hodgkiss, H. C. Song, T. Akal, C. Ferla, and D. R. Jackson, "Phase conjugation in the ocean: experimental demonstration of an acoustic time-reversal mirror," Journal of the Acoustical Society of America, vol. 103, no. 1, pp. 25-40, 1998.

[8] R. K. Ing, N. Quieffin, S. Catheline, and M. Fink, "In solid localization of finger impacts using acoustic time-reversal process," Applied Physics Letters, vol. 87, no. 20, Article ID 204104, 3 pages, 2005.

[9] M. Fink, G. Montaldo, and M. Tanter, "Time-reversal acoustics in biomedical engineering," Annual Review of Biomedical Engineering, vol. 5, pp. 465-497, 2003.

[10] G. Lerosey, J. de Rosny, A. Tourin, A. Derode, G. Montaldo, and M. Fink, "Time reversal of electromagnetic waves," Physical Review Letters, vol. 92, no. 19, pp. 193904-1, 2004.

[11] G. Lerosey, J. de Rosny, A. Tourin, A. Derode, and M. Fink, "Time reversal of wideband microwaves," Applied Physics Letters, vol. 88, no. 15, Article ID 154101, 2006.

[12] M. E. Yavuz and F. L. Teixeira, "Space-frequency ultrawideband time-reversal imaging," IEEE Transactions on Geoscience and Remote Sensing, vol. 46, no. 4, pp. 1115-1124, 2008.

[13] D. Liu, G. Kang, L. Li et al., "Electromagnetic time-reversal imaging of a target in a cluttered environment," IEEE Transactions on Antennas and Propagation, vol. 53, no. 9, pp. 30583066, 2005.

[14] J. M. F. Moura and Y. Jin, "Detection by time reversal: single antenna," IEEE Transactions on Signal Processing, vol. 55, no. 1, pp. 187-201, 2007.

[15] J. M. F. Moura and Y. Jin, “Time reversal imaging by adaptive interference canceling," IEEE Transactions on Signal Processing, vol. 56, no. 1, pp. 233-247, 2008.

[16] G. Lerosey, J. de Rosny, A. Tourin, A. Derode, G. Montaldo, and M. Fink, "Time reversal of electromagnetic waves and telecommunication," Radio Science, vol. 40, no. 6, Article ID RS6S12, 2005.

[17] A. Tourin, G. Lerosey, J. de Rosny, A. Derode, and M. Fink, "Time reversal telecommunications in complex environments," Comptes Rendus Physique, vol. 7, no. 7, pp. 816-822, 2006.

[18] R. C. Qiu, C. Zhou, N. Guo, and J. Q. Zhang, "Time reversal with MISO for ultrawideband communications: experimental results," IEEE Antennas and Wireless Propagation Letters, vol. 5, no. 1, pp. 269-273, 2006.

[19] T. Strohmer, M. Emami, J. Hansen, G. Papanicolaou, and A. J. Paulraj, "Application of time-reversal with MMSE equalizer to UWB communications," in Proceedings of the IEEE Global 
Telecommunications Conference (GLOBECOM '04), pp. 3123 3127, December 2004.

[20] P. Kosmas and C. M. Rappaport, "Time reversal with the FDTD method for microwave breast cancer detection," IEEE Transactions on Microwave Theory and Techniques, vol. 53, no. 7, pp. 2317-2323, 2005.

[21] B. Guo, L. Xu, and J. Li, "Time reversal based microwave hyperthermia treatment of breast cancer," in Proceedings of the 39th Asilomar Conference on Signals, Systems and Computers, pp. 290-293, November 2005.

[22] G. Lerosey, J. de Rosny, A. Tourin, and M. Fink, "Focusing beyond the diffraction limit with far-field time reversal," Science, vol. 315, no. 5815, pp. 1120-1122, 2007.

[23] M. Fink, J. de Rosny, G. Lerosey, and A. Tourin, "Timereversed waves and super-resolution," Comptes Rendus Physique, vol. 10, no. 5, pp. 447-463, 2009.

[24] J. de Rosny, G. Lerosey, and M. Fink, "Theory of electromagnetic time-reversal mirrors," IEEE Transactions on Antennas and Propagation, vol. 58, no. 10, pp. 3139-3149, 2010.

[25] G. Bartal, G. Lerosey, and X. Zhang, "Subwavelength dynamic focusing in plasmonic nanostructures using time reversal," Physical Review B, vol. 79, no. 20, Article ID 201103, 2009.

[26] A. Sentenac and P. C. Chaumet, "Subdiffraction light focusing on a grating substrate," Physical Review Letters, vol. 101, no. 1, Article ID 013901, 2008.

[27] D. Cassereau and M. Fink, "Time-reversal of ultrasonic fields-III: theory of the closed time-reversal cavity," IEEE Transactions on Ultrasonics, Ferroelectrics, and Frequency Control, vol. 39, no. 5, pp. 579-592, 1992.

[28] M. Fink, D. Cassereau, A. Derode et al., "Time-reversed acoustics," Reports on Progress in Physics, vol. 63, no. 12, pp. 19331995, 2000.

[29] I. M. Vellekoop and A. P. Mosk, "Focusing coherent light through opaque strongly scattering media," Optics Letters, vol. 32, no. 16, pp. 2309-2311, 2007.

[30] I. M. Vellekoop, A. Lagendijk, and A. P. Mosk, "Exploiting disorder for perfect focusing," Nature Photonics, vol. 4, pp. 320-322, 2010.

[31] S. M. Popoff, G. Lerosey, R. Carminati, M. Fink, A. C. Boccara, and S. Gigan, "Measuring the transmission matrix in optics: an approach to the study and control of light propagation in disordered media," Physical Review Letters, vol. 104, no. 10, Article ID 100601, 2010.

[32] G. Lerosey, Retournement temporel d'ondes électromagnétiques et application à la télécommunication en milieux complexes, Ph.D. thesis, Université Paris VII, 2006.

[33] R. Carminati, R. Pierrat, J. de Rosny, and M. Fink, "Theory of the time reversal cavity for electromagnetic fields," Optics Letters, vol. 32, no. 21, pp. 3107-3109, 2007.

[34] J. de Rosny and M. Fink, "Focusing properties of near-field time reversal," Physical Review A, vol. 76, no. 6, Article ID 065801, 2007.

[35] R. C. McPhedran, L. C. Botten, J. McOrist, A. A. Asatryan, C. M. de Sterke, and N. A. Nicorovici, "Density of states functions for photonic crystals," Physical Review E, vol. 69, no. 1, Article ID 016609, 16 pages, 2004.

[36] E. Synge, "A suggested method for extending microscopic resolution into the ultra-microscopic region," Philosophical Magazine, vol. 6, pp. 356-362, 1928.

[37] D. W. Pohl, W. Denk, and M. Lanz, "Optical stethoscopy: image recording with resolution $\lambda / 20$," Applied Physics Letters, vol. 44, no. 7, pp. 651-653, 1984.
[38] A. Lewis, M. Isaacson, A. Harootunian, and A. Muray, "Development of a $500 \AA$ spatial resolution light microscope. I. Light is efficiently transmitted through $\lambda / 16$ diameter apertures," Ultramicroscopy, vol. 13, no. 3, pp. 227-231, 1984.

[39] E. Betzig and J. K. Trautman, "Near-field optics: microscopy, spectroscopy, and surface modification beyond the diffraction limit," Science, vol. 257, no. 5067, pp. 189-195, 1992.

[40] F. Zenhausern, Y. Martin, and H. K. Wickramasinghe, "Scanning interferometric apertureless microscopy: optical imaging at 10 angstrom resolution," Science, vol. 269, no. 5227, pp. 1083-1085, 1995.

[41] R. Carminati, M. Nieto-Vesperinas, and J. J. Greffet, "Reciprocity of evanescent electromagnetic waves," Journal of the Optical Society of America A, vol. 15, no. 3, pp. 706-712, 1998.

[42] F. Lemoult, G. Lerosey, J. de Rosny, and M. Fink, "Resonant metalenses for breaking the diffraction barrier," Physical Review Letters, vol. 104, no. 20, Article ID 203901, 2010.

[43] P. A. Belov, Y. Hao, and S. Sudhakaran, "Subwavelength microwave imaging using an array of parallel conducting wires as a lens," Physical Review B, vol. 73, no. 3, pp. 1-4, 2006.

[44] G. Shvets, S. Trendafilov, J. B. Pendry, and A. Sarychev, "Guiding, focusing, and sensing on the subwavelength scale using metallic wire arrays," Physical Review Letters, vol. 99, no. 5, Article ID 053903, 2007.

[45] F. Goos and H. Hänchen, "Ein neuer und fundamentaler Versuch zur Totalreflexion," Annalen der Physik, vol. 436, no. 7-8, pp. 333-346, 1947.

[46] E. Purcell, "Spontaneous transition probabilities in radio-frequency spectroscopy," Physical Review, vol. 69, p. 681, 1946.

[47] A. Sarychev and V. Shalaev, Electrodynamics of Metamaterials, World Scientific, London, UK, 2007.

[48] D. R. Smith, W. J. Padilla, D. C. Vier, S. C. Nemat-Nasser, and S. Schultz, "Composite medium with simultaneously negative permeability and permittivity," Physical Review Letters, vol. 84, no. 18 , pp. 4184-4187, 2000.

[49] F. Capolino, Ed., Theory and Phenomena of Metamaterials, Taylor and Francis, London, UK, 2009.

[50] M. Tanter, J. L. Thomas, and M. Fink, "Time reversal and the inverse filter," Journal of the Acoustical Society of America, vol. 108, no. 1, pp. 223-234, 2000.

[51] G. Montaldo, M. Tanter, and M. Fink, "Real time inverse filter focusing through iterative time reversal," Journal of the Acoustical Society of America, vol. 115, no. 2, pp. 768-775, 2004.

[52] F. Lemoult, G. Lerosey, J. de Rosny, and M. Fink, "Manipulating spatiotemporal degrees of freedom of waves in random media," Physical Review Letters, vol. 103, no. 17, Article ID 173902, 2009.

[53] F. Lemoult, M. Fink, and G. Lerosey, "Acoustic resonators for far-field control of sound on a subwavelength scale," Physical Review Letters, vol. 107, no. 6, Article ID 064301, 2011. 

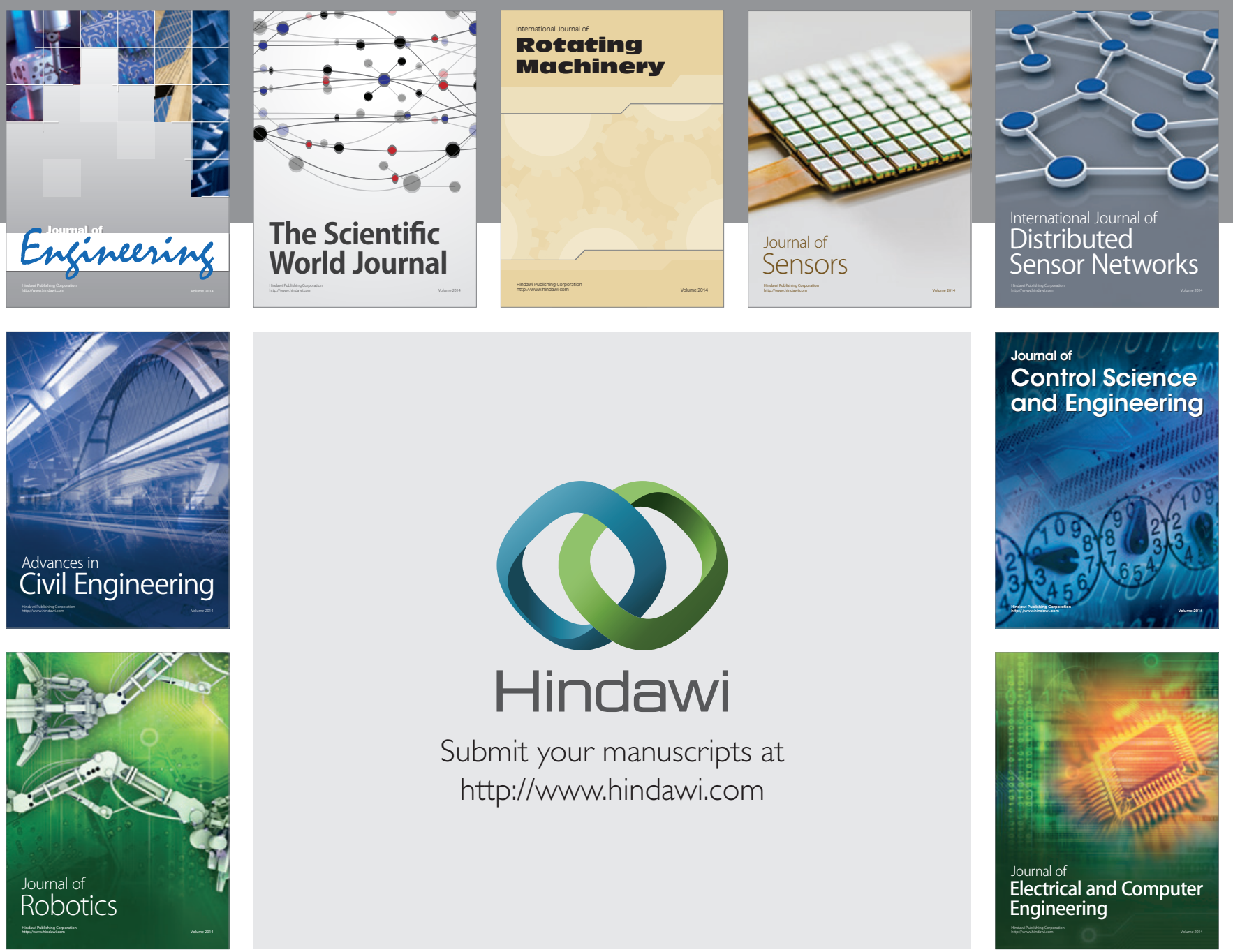

Submit your manuscripts at

http://www.hindawi.com
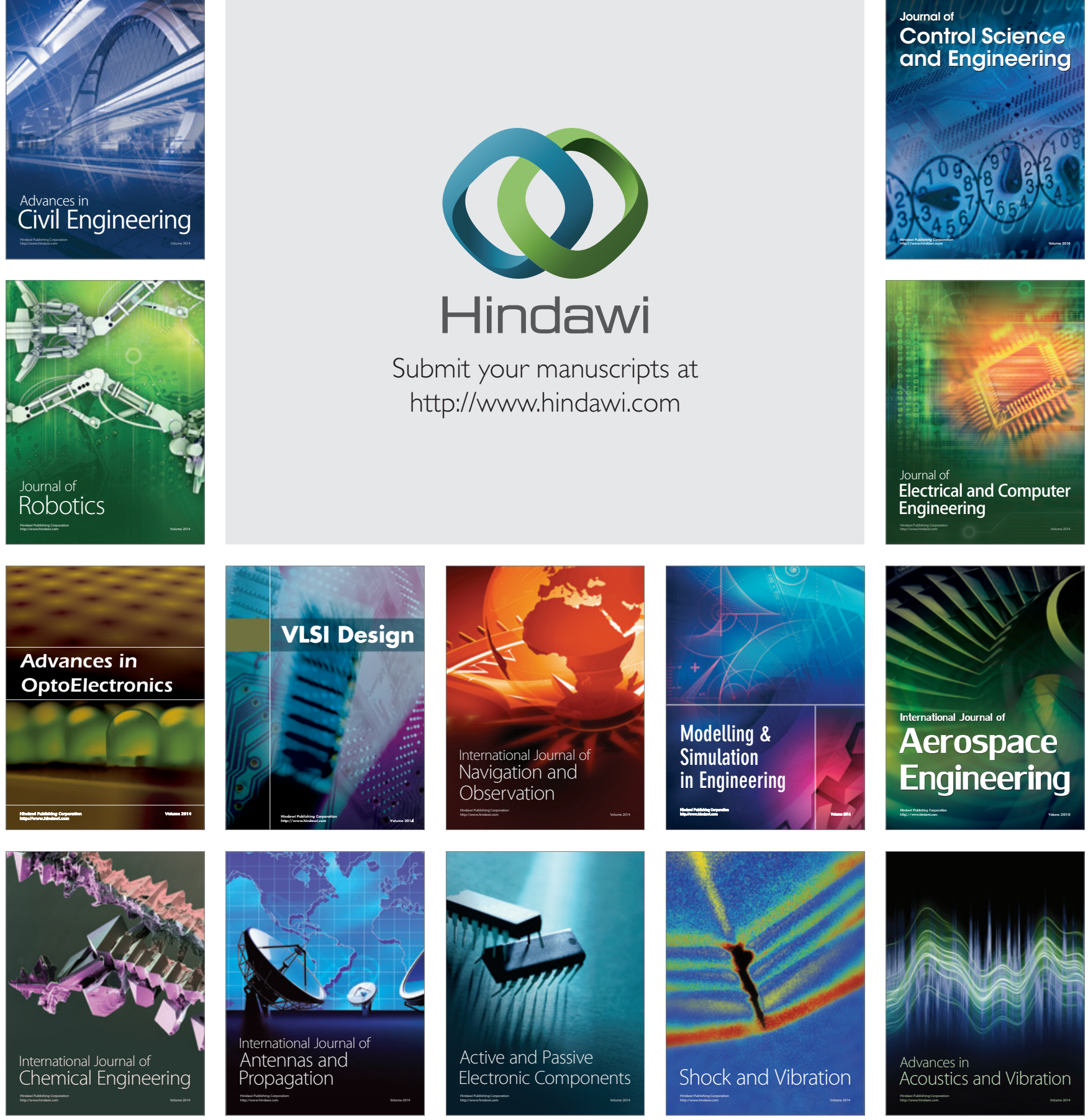